\title{
Hermitian Clifford analysis
}

\author{
I. Sabadini*and F. Sommen ${ }^{\dagger}$
}

\begin{abstract}
This paper gives an overview of some basic results on Hermitian Clifford analysis. It discusses, among other results, the Fischer decomposition, the Cauchy-Kowalewskaya extension problem, the axiomatic radial algebra and also some algebraic analysis of the system associated to Hermitian monogenic functions.
\end{abstract}

\section{Introduction}

Hermitian Clifford analysis is a refinement of classical Clifford analysis which is nowadays a very rich and well established field of research. It deals with functions with values in a complex Clifford algebra or a complex spinor space in the kernel of two mutually adjoint Dirac operators invariant under the action of the unitary group. Classical Clifford analysis, see [27, 37] deals with functions in the kernel of the Dirac operator, which is invariant under the action of the special orthogonal group and for this reason it may be called orthogonal Clifford analysis. It is important to point out that by restricting the values of a Hermitian monogenic function one obtains functions holomorphic in several complex variables.

The results available in the literature are numerous and it is not possible to mention all of them in this survey whose contents just reflects some basics facts and the authors' taste.

Although the systems presented in [45] are related, the study of the Hermitian monogenic systems in its present form began in [46] and the fundamentals of the theory have been further developed in $[8,9,25]$. Then, just to give an overview of the results, one has to mention that the Cauchy formula with several questions related to it has been studied in $[1,3,5,13]$, while the Hilbert transform has been treated in $[2,10,12]$. The Cauchy-Kovalevskaya (or CK) extension theorem has been considered in $[16,35]$, but the complete treatment of the CK-extension problem requires the introduction of a weaker system called the submonogenic system (see [36]). Papers dealing with wavelets are $[16,22,24]$. The important topic of the Fischer decomposition and related questions has been addressed in $[25,26,38,40]$. Questions related to polynomial bases can be found in [17, 21] and Taylor series expansion are in [42]. The algebraic analysis of $h$-hermitian functions has been done in $[46,28,32,33]$ and some duality theorems are proved in [7, 29]. To conclude this introduction, it is worthwhile to mention a generalization of Hermitian Clifford analysis over the quaternions, see [44]. This generalization is introduced and studied with a different approach in [39] and an algebraic analysis of the system has been performed in [34].

The plan of this survey is as follows. Section 2 provides the preliminaries which are necessary to introduce the Witt basis, then in Section 3 the Hermitian vector variables, the Dirac operators and the notion of Hermitian monogenic functions are defined. The CK-extension problem is

\footnotetext{
*Politecnico di Milano, Dipartimento di Matematica, Via Bonardi 9, 20133 Milano, Italy, irene.sabadini@polimi.it

${ }^{\dagger}$ Clifford Research Group, Faculty of Sciences, Ghent University, Galglaan 2, 9000 Gent, Belgium, fs@cage.ugent.be
} 
treated in Section 3. Section 4 discusses the Fischer decomposition, while Section 5 deals with some integral formulae, among which the Cauchy integral formula. Section 6 contains the axiomatic definition of the hermitian radial algebra and the survey ends by presenting, in Section 7 , the algebraic analysis of the module associated to $h$-hermitian functions.

\section{The Clifford algebra setting}

Consider the real orthogonal space $\mathbb{R}^{2 n}=\operatorname{span}_{\mathbb{R}}\left(e_{1}, \cdots, e_{2 n}\right)$ endowed with the symmetric realbilinear form $\mathcal{B}_{\mathbb{R}}(\cdot, \cdot)$ of signature $(0,2 n)$, for which $\mathcal{B}_{\mathbb{R}}\left(e_{i}, e_{j}\right)=-\delta_{i j}$. At the same time, one also considers the complex vector space $\mathbb{C}^{2 n}$ and its associated Clifford algebra $\mathbb{C}_{2 n}$, generated by $e_{i} e_{j}+e_{j} e_{i}=-2 \delta_{i j}$. A basis for $\mathbb{C}_{2 n}$ can be constructed by considering, for any set of indices $A=\left\{j_{1}, \ldots, j_{h}\right\} \subset\{1, \ldots, 2 n\}=M$, ordered by $1 \leq j_{1}<j_{2}<\ldots<j_{h} \leq 2 n$, the element $e_{A}=e_{j_{1}} \ldots e_{j_{h}}$ where $e_{\emptyset}=1$, the identity element. Any Clifford number $\lambda \in \mathbb{C}_{2 n}$ may thus be written as

$$
\lambda=\sum_{A \subset M} \lambda_{A} e_{A}, \quad \lambda_{A} \in \mathbb{C} .
$$

One can also write $\lambda=\sum_{k=0}^{2 n}[\lambda]_{k}$ where $[\lambda]_{k}=\sum_{|A|=k} \lambda_{A} e_{A}$ is the so-called $k$-vector part of $\lambda(k=0, \ldots, 2 n)$.

Denoting by $\mathbb{C}_{2 n}^{k}$ the subspace of all $k$-vectors in $\mathbb{C}_{2 n}$, then $\mathbb{C}$ can be identified with the subspace of complex scalars $\mathbb{C}_{2 n}^{0}$, while $\mathbb{R}^{2 n}$ can be identified with the subspace of real Clifford vectors $\mathbb{R}_{0,2 n}^{1}=\left\{\underline{v}=\sum_{j=1}^{2 n} v_{j} e_{j}, v_{j} \in \mathbb{R}\right\} \quad \subset \quad \mathbb{C}_{2 n}^{1}$. It is also important to note that all real Clifford algebras $\mathbb{R}_{p, q}$ with signature $(p, q)(p+q=2 n)$ are contained in $\mathbb{C}_{2 n}$ as special subalgebras.

In $\mathbb{C}_{2 n}$ there automorphisms which leave the multivector structure invariant:

1. the main involution

$$
\widetilde{\lambda \mu}=\widetilde{\lambda} \widetilde{\mu} ; \widetilde{\mu_{A} e_{A}}=\mu_{A} \widetilde{e_{A}} ; \quad \widetilde{e_{j}}=-e_{j}(j=1, \ldots, 2 n)
$$

2. the reversion

$$
(\lambda \mu)^{*}=\mu^{*} \lambda^{*} ; \quad\left(\mu_{A} e_{A}\right)^{*}=\mu_{A} e_{A}^{*}(A \subset M) ; \quad e_{j}^{*}=e_{j}(j=1, \ldots, 2 n)
$$

3. the Hermitian conjugation (or $h$-conjugation)

$$
(\lambda \mu)^{\dagger}=\mu^{\dagger} \lambda^{\dagger} ; \quad\left(\mu_{A} e_{A}\right)^{\dagger}=\mu_{A}^{c} e_{A}^{\dagger}(A \subset M) ; \quad e_{j}^{\dagger}=-e_{j}(j=1, \ldots, 2 n)
$$

where $\mu_{A}^{c}$ stands for the complex conjugate of the complex number $\mu_{A}$.

By restricting the $h$-conjugation to the real subalgebra $\mathbb{R}_{0,2 n}(p=0, q=2 n)$ one gets

$$
e_{j}^{\dagger}=-e_{j}, \quad j=1, \ldots, 2 n
$$

and

$$
\left(a_{A} e_{A}\right)^{\dagger}=a_{A} e_{A}^{\dagger}, \quad a_{A} \in \mathbb{R},
$$

so this restriction coincides with the conjugation on $\mathbb{R}_{0,2 n}$ i.e.

$$
\overline{(a b)}=\bar{b} \bar{a} ; \quad \overline{e_{j}}=-e_{j}, j=1, \ldots, 2 n .
$$


For the real subalgebras $\mathbb{R}_{p, q}, p \neq 0$, the situation is different since for $j=1, \ldots, p$ it is $\varepsilon_{j}^{\dagger}=\left(i e_{j}\right)^{\dagger}=i e_{j}=\varepsilon_{j}$. Since $\mathbb{C}_{2 n}$ may be regarded as the complexification of the real Clifford algebra $\mathbb{R}_{0,2 n}$, namely

$$
\mathbb{C}_{2 n}=\mathbb{C} \otimes \mathbb{R}_{0,2 n}=\mathbb{R}_{0,2 n} \oplus i \mathbb{R}_{0,2 n}
$$

any element $\lambda \in \mathbb{C}_{2 n}$ can be written as $\lambda=a+i b, a \in \mathbb{R}_{0,2 n}, b \in \mathbb{R}_{0,2 n}$. Moreover

$$
\lambda^{\dagger}=(a+i b)^{\dagger}=a^{\dagger}-i b^{\dagger}=\bar{a}-i \bar{b}
$$

Using the $h$-conjugation one can define an Hermitian inner product, called $h$-inner product, whose associated $h$-norm on $\mathbb{C}_{2 n}$ is

$$
(\lambda, \mu)=\left[\lambda^{\dagger} \mu\right]_{0}, \quad|\lambda|=\sqrt{\left[\lambda^{\dagger} \lambda\right]_{0}} .
$$

Note that for complex Clifford vectors $\underline{\alpha}=\sum_{j=1}^{2 n} \alpha_{j} e_{j}$ and $\underline{\beta}=\sum_{j=1}^{2 n} \beta_{j} e_{j}$ their $h$-inner product reduces to

$$
(\underline{\alpha}, \underline{\beta})=\sum_{j=1}^{m} \alpha_{j}^{c} \beta_{j}
$$

and $|\underline{\alpha}|^{2}=(\underline{\alpha}, \underline{\alpha})=\sum_{j=1}^{2 n}\left|\alpha_{j}\right|^{2}$.

A main tool in Hermitian Clifford analysis, namely the Witt basis, is defined below.

Definition 1. Let us consider in $\mathbb{C}_{2 n}$ the elements

$$
\begin{aligned}
\mathfrak{f}_{j} & =\frac{1}{2}\left(e_{j}-i e_{n+j}\right), & j & =1, \ldots, n \\
\mathfrak{f}_{j}^{\dagger} & =-\frac{1}{2}\left(e_{j}+i e_{n+j}\right), & j & =1, \ldots, n .
\end{aligned}
$$

These elements form the so-called Witt basis of $\mathbb{C}_{2 n}$; they satisfy the Grassmann and duality identities

(i) $\mathfrak{f}_{j} \mathfrak{f}_{k}+\mathfrak{f}_{k} \mathfrak{f}_{j}=0$,

(ii) $\mathfrak{f}_{j}^{\dagger} \mathfrak{f}_{k}^{\dagger}+\mathfrak{f}_{k}^{\dagger} \mathfrak{f}_{j}^{\dagger}=0$,

(iii) $\mathfrak{f}_{j} \mathfrak{f}_{k}^{\dagger}+\mathfrak{f}_{k}^{\dagger} \mathfrak{f}_{j}=\delta_{j k}$,

for $j, k=1, \ldots, n$.

Remark 1. From these relations one obtains:

$$
\mathfrak{f}_{j}^{2}=\left(\mathfrak{f}_{j}^{\dagger}\right)^{2}=0 .
$$

Note also that $\mathfrak{f}_{j}^{\dagger}$ is the $h$-conjugate of $\mathfrak{f}_{j}, j=1, \ldots, n$.

The above relations may be refined in a series of properties for their dot and wedge products, and their $h$-inner product, see [25].

The above construction can be done in an alternative way, see [9], [8]. Consider the real orthogonal space $\mathbb{R}^{2 n}=\operatorname{span}_{\mathbb{R}}\left(e_{1}, \cdots, e_{2 n}\right)$ equipped with the symmetric real-bilinear form $\mathcal{B}_{\mathbb{R}}(\cdot, \cdot)$ of signature $(0,2 n)$, for which $\mathcal{B}_{\mathbb{R}}\left(e_{i}, e_{j}\right)=-\delta_{i j}$. Then consider the complex vector space $\mathbb{C}^{2 n}$ and its associated Clifford algebra $\mathbb{C}_{2 n}$, generated by $e_{i} e_{j}+e_{j} e_{i}=-2 \delta_{i j}$. It 
is immediate that, as a vector space, $\mathbb{C}_{2 n}$ is isomorphic to a Grassmann algebra and hence inherits a $\mathbb{Z}$-grading into subspaces $\mathbb{C}_{2 n}^{(k)}$ of $k$-vectors. On the other hand, $\mathbb{C}_{2 n}$ is $\mathbb{Z}_{2}$-graded with respect to the main involution, acting on arbitrary Clifford numbers as $\widetilde{(a b)}=\widetilde{a} \widetilde{b}$. As a consequence one can decompose $\mathbb{C}_{2 n}=\mathbb{C}_{2 n}^{+} \oplus \mathbb{C}_{2 n}^{-}$into a direct sum of even and odd elements. Within $\mathbb{C}_{2 n}^{+}$, one can define the (real or complex) spin group $\operatorname{Spin}(2 n)$ which yields a double cover for the (real or complex) group $S O(2 n)$. The real spin group $\operatorname{Spin}(2 n, \mathbb{R})$ can be realized within the Clifford algebra as

$$
\operatorname{Spin}(2 n, \mathbb{R})=\left\{s=\prod_{j=1}^{2 k} \underline{\omega}_{j}: \underline{\omega}_{j} \in S^{2 n-1}, k \in \mathbb{N}\right\},
$$

where $S^{2 n-1} \subset \mathbb{R}^{2 n}$ denotes the unit sphere containing unit vectors $\underline{\omega}_{j}$ for which $\underline{\omega}_{j}^{2}=-1$. The double cover between both Lie groups is then defined by the mapping $h: \operatorname{Spin}(2 n, \mathbb{R}) \mapsto$ $S O(2 n, \mathbb{R})$ for which $h(s)[\underline{X}]=s \underline{X} \bar{s}$ where the main conjugation acts on arbitrary Clifford numbers by $\overline{a b}=\bar{b} \bar{a}$.

Definition 2. A complex structure $J$ on $\mathbb{R}^{2 n}$ can be defined as an automorphism $J \in S O(2 n, \mathbb{R})$ satisfying $J^{2}=-\mathbf{1}_{2 n}$.

Remark 2. In terms of the basis $\left(e_{1}, \cdots, e_{2 n}\right)$ one may put $J\left[e_{j}\right]=-e_{j+n}$ and $J\left[e_{j+n}\right]=+e_{j}$. By means of the complex structure $J$, one can decompose $\mathbb{C}^{2 n}$, endowed with the complexbilinear form $\mathcal{B}_{\mathbb{C}}$, into a direct sum of two maximally isotropic subspaces $W^{+}$and $W^{-}$. Put

$$
\mathbf{1}_{2 n}=\frac{1}{2}\left(\mathbf{1}_{2 n}+i J\right)+\frac{1}{2}\left(\mathbf{1}_{2 n}-i J\right)=\pi^{+}+\pi^{-},
$$

and define $W^{ \pm}=\pi^{ \pm}\left(\mathbb{R}^{2 n}\right)$. The spaces $W^{ \pm}$are isotropic, in the sense that the restrictions of $\mathcal{B}_{\mathbb{C}}$ to $W^{+} \times W^{+}$and $W^{-} \times W^{-}$are identically zero, and that $W^{ \pm}$are eigenspaces for the complex-linear map $J_{\mathbb{C}} \in S O(2 n)$ with eigenvalues $\mp i$.

The Witt basis for $\mathbb{C}^{2 n}$ is then defined by

$$
\mathfrak{f}_{j}=+\pi^{+}\left[e_{j}\right], \quad \mathfrak{f}_{j}^{\dagger}=-\pi^{-}\left[e_{j}\right], \quad 1 \leq j \leq n .
$$

Using the Witt basis elements $\left(\mathfrak{f}_{1}, \ldots, \mathfrak{f}_{n}\right)$ and $\left(\mathfrak{f}_{1}^{\dagger}, \ldots, \mathfrak{f}_{n}^{\dagger}\right)$ of $\mathbb{C}_{2 n}$, one can introduce the complex Grassmann algebras $\mathbb{C} \Lambda_{n}$ and $\mathbb{C} \Lambda_{n}^{+}$defined by:

$$
\mathbb{C} \Lambda_{n}=\operatorname{Alg}_{\mathbb{C}}\left\{\mathfrak{f}_{1}, \ldots, \mathfrak{f}_{n}\right\} \quad \text { and } \quad \mathbb{C} \Lambda_{n}^{\dagger}=\operatorname{Alg}_{\mathbb{C}}\left\{\mathfrak{f}_{1}^{\dagger}, \ldots, \mathfrak{f}_{n}^{\dagger}\right\} .
$$

In particular, set

$$
\mathbb{C} \Lambda_{n}^{k}=\mathbb{C}_{2 n}^{k} \cap \mathbb{C} \Lambda_{n}, \quad \mathbb{C} \Lambda_{n}^{\dagger}=\mathbb{C}_{2 n}^{k} \cap \mathbb{C} \Lambda_{n}^{\dagger} .
$$

Let us now introduce, for all $j=1, \ldots, n$, the elements

$$
I_{j}=\mathfrak{f}_{j} \mathfrak{f}_{j}^{\dagger}=\frac{1}{2}\left(1+i e_{n+j} e_{j}\right)=\frac{1}{2}\left(1-i e_{j} e_{n+j}\right) .
$$

The $I_{j}$ 's are mutually commuting idempotents, i.e. $I_{j}^{2}=I_{j}$ and $I_{j} I_{k}=I_{k} I_{j}$, moreover $I_{j}^{\dagger}=I_{j}$. Now put

$$
I=I_{1} \ldots I_{n} .
$$

Then $I^{\dagger}=I$ and

$$
\begin{aligned}
& e_{j} I=i e_{n+j} I=-\mathfrak{f}_{j}^{\dagger} I, \quad \mathfrak{f}_{j} I=0, \quad j=1, \ldots, n \\
& I e_{j}=-i I e_{n+j}=I \mathfrak{f}_{j}, \quad I \mathfrak{f}_{j}^{\dagger}=0, \quad j=1, \ldots, n \text {. }
\end{aligned}
$$

One also has 
Proposition 1. A vector $\underline{\alpha} \in \mathbb{C}_{2 n}$ belongs to $\mathbb{C} \Lambda_{n}^{1}$ if and only if $\underline{\alpha} I=0$, while it belongs to $\mathbb{C} \Lambda_{n}^{\dagger}{ }^{1}$ if and only if $I \underline{\alpha}=0$.

Definition 3. The complex spinor space $\mathbb{C S}_{n}$ is defined as

$$
\mathbb{C S}_{n} \equiv \mathbb{C}_{2 n} I \cong \mathbb{C}_{n} I \cong \mathbb{C} \Lambda_{n}^{\dagger} I
$$

and its homogeneous parts are

$$
\mathbb{C S}_{n}^{k} \equiv \mathbb{C}_{n}^{k} I \cong \mathbb{C} \Lambda_{n}^{\dagger} I \quad\left(\neq \quad \mathbb{C}_{2 n}^{k} I\right)
$$

Finally, note that by the isomorphisms established above, for any $a \in \mathbb{C}_{2 n}$ (or $\mathbb{R}_{n, n}$ ), there exists a unique element $\widehat{a} \in \mathbb{C}_{n}$ (or $\mathbb{R}_{0, n}$ ) such that $a I=\widehat{a} I$, and thus also

$$
I a I=I \widehat{a} I=I[\widehat{a}]_{0} I=[\widehat{a}]_{0} I^{2}=[\widehat{a}]_{0} I
$$

since for all $k$-vector parts of $\widehat{a}$ one has that $I[\widehat{a}]_{k} I=0, k=1, \ldots, n$.

\subsection{The spin group}

Definition 4. Let us define the complex spin group in the Clifford algebra $\mathbb{C}_{2 n}$ as

$$
\operatorname{Spin}(2 n, \mathbb{C})=\mathbb{C} \otimes \operatorname{Spin}(2 n, \mathbb{R}),
$$

where

$$
\operatorname{Spin}(2 n, \mathbb{R}) \subseteq\left\{s \in \mathbb{R}_{0,2 n} \mid s \bar{s}=\bar{s} s=1\right\} .
$$

As it is well known that any element $s$ of $\operatorname{Spin}(2 n, \mathbb{R})$ may be written as

$$
s=\underline{\omega}_{1} \ldots \underline{\omega}_{2 l}, \quad \underline{\omega}_{j} \in \mathbb{R}_{0,2 n}^{1}, \quad \underline{\omega}_{j}^{2}=-1, \quad j=1, \ldots, 2 l, l \in \mathbb{N}
$$

It is of interest the subgroup $\widetilde{\mathrm{U}}(n)$, given by

$$
\widetilde{\mathrm{U}}(n)=\{s \in \operatorname{Spin}(2 n) \mid \exists \theta \geq 0: \bar{s} I=\exp (-i \theta) I\}
$$

which is a representation of the unitary group $\mathrm{U}(n)$. It is important to note that for any $s \in \operatorname{Spin}(2 n)$ it is $s \bar{s}=\bar{s} s=1$, and since $s^{\dagger}=\bar{s}=s^{-1}$, one deduces the relation $I s=\exp (i \theta) I$ for $s \in \widetilde{\mathrm{U}}(n)$ (this relation is the $h$-conjugate of the one contained in (2.1)).

Since $\operatorname{Spin}(2 n, \mathbb{R})$ is a double covering of $S O(2 n, \mathbb{R})$, denote by $s_{J}$ the element in $\operatorname{Spin}(2 n, \mathbb{R})$ corresponding to the complex structure $J$ and define

$$
\operatorname{Spin}_{J}(2 n, \mathbb{R})=\left\{s \in \operatorname{Spin}(2 n, \mathbb{R}): s s_{J}=s_{J} s\right\} .
$$

Then the following result holds (see [9]):

Proposition 2. The groups $\operatorname{Spin}_{J}(2 n, \mathbb{R})$ and $\widetilde{U}(n)$ coincide. 


\section{The Hermitian operators}

\subsection{Hermitian vector variables and Dirac operators}

Let us introduce the Hermitian vector variables, also called $h$-vector variables for short. First of all, the vector $\left(X_{1}, \ldots, X_{2 n}\right)=\left(x_{1}, \ldots, x_{n}, y_{1}, \ldots, y_{n}\right)$ can be identified with the Clifford vector

$$
\underline{X}=\sum_{j=1}^{n} e_{j} X_{j}=\sum_{j=1}^{n}\left(e_{j} x_{j}+e_{n+j} y_{j}\right)
$$

In terms of the Witt basis it can be written as

$$
\underline{X}=\sum_{j=1}^{n}\left(\left(x_{j}+i y_{j}\right) \mathfrak{f}_{j}-\left(x_{j}-i y_{j}\right) \mathfrak{f}_{j}^{\dagger}\right)=\sum_{j=1}^{n} \mathfrak{f}_{j} z_{j}-\sum_{j=1}^{n} \mathfrak{f}_{j}^{\dagger} z_{j}^{c}
$$

where $z_{j}=x_{j}+i y_{j}$ are complex variables and their complex conjugates are $z_{j}^{c}=x_{j}-i y_{j}$, $j=1, \ldots, n$. The Hermitian vector variable, also called $h$-vector variables, are defined as

$$
\underline{z}=\sum_{j=1}^{n} \mathfrak{f}_{j} z_{j}
$$

with $h$-conjugate

$$
\underline{z}^{\dagger}=\sum_{j=1}^{n} \mathfrak{f}_{j}^{\dagger} z_{j}^{c}
$$

Thus the Clifford vector $\underline{X}$ can be written as

$$
\underline{X}=\underline{z}-\underline{z}^{\dagger}
$$

Another Clifford vector (which is called the twist of $\underline{X}$ ) is defined as

$$
\underline{X} \mid=\frac{1}{i}\left(\underline{z}+\underline{z}^{\dagger}\right)=\frac{1}{i} \sum_{j=1}^{n}\left(z_{j} \mathfrak{f}_{j}+z_{j}^{c} \mathfrak{f}_{j}^{\dagger}\right)=\sum_{j=1}^{n}\left(y_{j} e_{j}-x_{j} e_{n+j}\right) ;
$$

note that $\underline{X}$ and $\underline{X} \mid$ are orthogonal:

$$
(\underline{X}, \underline{X} \mid)=\sum_{j=1}^{n} x_{j} y_{j}+\sum_{j=1}^{n} y_{j}\left(-x_{j}\right)=0 .
$$

Remark 3. Observe that the equalities $\underline{z}^{2}=\left(\underline{z}^{\dagger}\right)^{2}=0$ hold. For other relations on the $h$-vector variables, see [25].

Let us now introduce suitable differential operators. The Dirac operator associated to $\underline{X}$ is:

$$
\partial_{\underline{X}}=\sum_{j=1}^{n}\left(e_{j} \partial_{x_{j}}+e_{n+j} \partial_{y_{j}}\right)=\sum_{j=1}^{n}\left(\mathfrak{f}_{j}\left(\partial_{x_{j}}+i \partial_{y_{j}}\right)-\mathfrak{f}_{j}^{\dagger}\left(\partial_{x_{j}}-i \partial_{y_{j}}\right)\right),
$$

and the Cauchy-Riemann operators (and their conjugates) in the complex variables $z_{j},(j=$ $1, \ldots, n)$ are:

$$
\partial_{z_{j}}=\frac{1}{2}\left(\partial_{x_{j}}-i \partial_{y_{j}}\right) \quad \text { and } \quad \partial_{z_{j}^{c}}=\frac{1}{2}\left(\partial_{x_{j}}+i \partial_{y_{j}}\right) .
$$

Then the Dirac operator can be written as

$$
\partial_{\underline{X}}=2 \sum_{j=1}^{n}\left(\mathfrak{f}_{j} \partial_{z_{j}^{c}}-\mathfrak{f}_{j}^{\dagger} \partial_{z_{j}}\right)=2\left(\partial_{\underline{z}}^{\dagger}-\partial_{\underline{z}}\right) .
$$


Definition 5. The Hermitian (or $h$-)Dirac operator, also called $h$-Dirac operator and its $h$-conjugate are defined by

$$
\partial_{\underline{z}}=\sum_{j=1}^{n} \mathfrak{f}_{j}^{\dagger} \partial_{z_{j}} \quad \text { and } \quad \partial_{\underline{z}}^{\dagger}=\left(\partial_{\underline{z}}\right)^{\dagger}=\sum_{j=1}^{n} \mathfrak{f}_{j} \partial_{z_{j}^{c}} .
$$

Remark 4. Note that $\partial_{\underline{z}}^{\dagger}=\partial_{\underline{z}^{\dagger}}$ and both the notations are used in the literature.

To the Clifford vector variable $\underline{X} \mid$ one can associate the following operator

$$
\partial_{\underline{X} \mid}=\sum_{j=1}^{n}\left(e_{j} \partial_{y_{j}}-e_{n+j} \partial_{x_{j}}\right)=\frac{2}{i} \sum_{j=1}^{n}\left(\mathfrak{f}_{j} \partial_{z_{j}^{c}}+\mathfrak{f}_{j}^{\dagger} \partial_{z_{j}}\right)=\frac{2}{i}\left(\partial_{\underline{z}}^{\dagger}+\partial_{\underline{z}}\right)
$$

Remark 5. With the notation introduced in Remark 2 one has

$$
2 \partial_{\underline{z}}^{\dagger}=+\pi^{+}\left[\partial_{\underline{X}}\right]=2 \sum_{j=1}^{n} \mathfrak{f}_{j} \partial_{z_{j}^{c}} \quad 2 \partial_{\underline{z}}=-\pi^{-}\left[\partial_{\underline{X}}\right]=2 \sum_{j=1}^{n} \mathfrak{f}_{j}^{\dagger} \partial_{z_{j}} .
$$

In order to give the definition of Hermitian monogenic functions, recall that the functions $g$ defined on $\mathbb{R}^{2 n}$, may be written as

$$
g\left(x_{1}, \ldots, x_{n}, y_{1}, \ldots, y_{n}\right), g\left(z_{1}, \ldots, z_{n}, z_{1}^{c}, \ldots, z_{n}^{c}\right) \text { or, in short } g\left(\underline{z}, \underline{z}^{\dagger}\right)
$$

and taking values in the complex Clifford algebra $\mathbb{C}_{2 n}$.

Definition 6. Let $\Omega$ be an open region in $\mathbb{R}^{2 n}$ and let $f: \Omega \rightarrow \mathbb{C}_{2 n}$ be a continuously differentiable function. We say that $g$ is Hermitian monogenic, or $h$-monogenic for short, if and only if

$$
\partial_{\underline{X}} g=0=\partial_{\underline{X}} g
$$

or, equivalently,

$$
\partial_{\underline{z}} g=0=\partial_{\underline{z}}^{\dagger} g \text {. }
$$

Example 1. Any function of the form $g_{j}=\mathfrak{f}_{j}^{\dagger} F_{j}\left(z_{j}\right), j=1, \ldots, n$, with $F_{j}$ holomorphic in the open region $\Omega_{j}$ of the complex $z_{j}$ plane, is $h$-monogenic in $\Omega=\mathbb{R}^{2} \times \ldots \times \mathbb{R}^{2} \times \Omega_{j} \times \mathbb{R}^{2} \times \ldots \times \mathbb{R}^{2}$. In fact

$$
\partial_{\underline{z}} g_{j}=\mathfrak{f}_{j}^{\dagger} \mathfrak{f}_{j}^{\dagger} \partial_{z_{j}} F_{j}\left(z_{j}\right)=0
$$

since $\left(\mathfrak{f}_{j}^{\dagger}\right)^{2}=0$, while

$$
\partial_{\underline{z}}^{\dagger} g_{j}=\mathfrak{f}_{j} \dagger_{j}^{\dagger} \partial_{z_{j}^{c}} F_{j}\left(z_{j}\right)=0 .
$$

Any function of the form $\widetilde{g}_{j}=\mathfrak{f}_{j} \widetilde{F}_{j}\left(z_{j}^{c}\right), j=1, \ldots, n$, with $\widetilde{F}_{j}$ anti-holomorphic in the open region $\Omega_{j}$, is $h$-monogenic in $\Omega$.

Remark 6. If a function $g$ has values in $\mathbb{C}_{n}$, the above system may be written, equivalently, by considering the spinor valued function $g I$ which has values in $\mathbb{C}_{n} I \cong \mathbb{C}_{2 n} I=\mathbb{C S}_{n}$ :

$$
\partial_{\underline{X}}(g I)=0=\partial_{\underline{X}}(g I) \quad \text { or, equivalently, } \quad \partial_{\underline{z}}(g I)=0=\partial_{\underline{z}}^{\dagger}(g I) .
$$


Since $\varepsilon_{j} g I=\widetilde{g} e_{j} I, j=1, \ldots, n$,

$$
\begin{aligned}
\partial_{\underline{X}}(g I) & =\sum_{j=1}^{n}\left(e_{j} \partial_{x_{j}}-i \varepsilon_{j} \partial_{y_{j}}\right)(g I) \\
& =\sum_{j=1}^{n} e_{j}\left(\partial_{x_{j}} g\right) I-i \sum_{j=1}^{n}\left(\partial_{y_{j}} \widetilde{g}\right) e_{j} I=\left(\partial_{\underline{x}} g-i \widetilde{g} \partial_{\underline{y}}\right) I
\end{aligned}
$$

where $\partial_{\underline{x}}=\sum_{j=1}^{n} e_{j} \partial_{x_{j}}$ and $\partial_{\underline{y}}=\sum_{j=1}^{n} e_{j} \partial_{y_{j}}$. Similarly,

$$
\partial_{\underline{X}} \mid(g I)=\left(g \partial_{\underline{x}}-i \partial_{\underline{y}} \widetilde{g}\right) I .
$$

This gives an alternative formulation of the condition of $h$-monogenicity for the subclass of functions having values in $\mathbb{C}_{n}$ :

Proposition 3. A function $g: \mathbb{R}^{2 n} \rightarrow \mathbb{C}_{n}$ is $h-$ monogenic if and only if

$$
\partial_{\underline{x}} g-i \widetilde{g} \partial_{\underline{y}}=0=g \partial_{\underline{x}}-i \partial_{\underline{y}} \tilde{g}
$$

If a function $g_{k}: \mathbb{R}^{2 n} \rightarrow \mathbb{C}_{n}^{k}$ is pure $k$-vector valued, then $g_{k}$ is $h$-monogenic if and only if

$$
\partial_{\underline{X}}\left(g_{k} I\right)=0
$$

since for such a function $\partial_{\underline{X}}\left(g_{k} I\right)=0$ implies $\partial_{\underline{X}}\left(g_{k} I\right)=0$ by Proposition 3 .

Conversely:

Proposition 4. A function $g: \mathbb{R}^{2 n} \rightarrow \mathbb{C}_{n}$ is $h-$ monogenic if and only if

$$
\partial_{\underline{X}}\left([g]_{k} I\right)=0, \quad k=0, \ldots, n
$$

where $[g]_{k}$ denotes the projection of $g$ onto the space of $k$-vectors.

Remark 7. There is a relation between the $h$-Dirac operators and the Laplace operator, specifically:

$$
-\Delta_{2 n}=\partial_{\underline{X}}^{2}=4\left(\partial_{\underline{z}}^{\dagger}-\partial_{\underline{z}}\right)^{2}=(-4)\left(\partial_{\underline{z}}^{\dagger} \partial_{\underline{z}}+\partial_{\underline{z}} \partial_{\underline{z}}^{\dagger}\right)
$$

since $\partial_{\underline{z}} \partial_{\underline{z}}=\partial_{\underline{z}}^{\dagger} \partial_{\underline{z}}^{\dagger}=0$. Analogously:

$$
-\Delta_{2 n}=\partial_{\underline{X} \mid}^{2}=(-4)\left(\partial_{\underline{z}}^{\dagger}+\partial_{\underline{z}}\right)^{2} .
$$

It follows that $h-$ monogenicity implies harmonicity. Moreover

$$
\partial_{\underline{z}} \cdot \partial_{\underline{z}}^{\dagger}=\partial_{\underline{z}}^{\dagger} \cdot \partial_{\underline{z}}=\frac{1}{2} \Delta_{2 n} \quad \text { and } \quad \partial_{\underline{z}} \partial_{\underline{z}}^{\dagger}-\partial_{\underline{z}}^{\dagger} \partial_{\underline{z}}=2 \partial_{\underline{z}} \wedge \partial_{\underline{z}}^{\dagger}
$$

An important consequence of the above is the following (see [8]):

Proposition 5. Let $g=\left(g_{0}, \ldots, g_{n}\right)$ be the decomposition of $g: \mathbb{C}^{2 n} \rightarrow \mathbb{C S}_{n}$ into its homogeneous spinor parts, i.e. $g_{j} \in \mathbb{C S}_{n}^{j}, j=0, \ldots, n$. Then the system $\partial_{\underline{z}} g=0$ is equivalent to the systems $\partial_{\underline{z}} g_{j}=0, j=0, \ldots, n$. The system $\partial_{\underline{z}}^{\dagger} g=0$ is equivalent to the systems $\partial_{\underline{z}}^{\dagger} g_{j}=0$, $j=0, \ldots, n$. 


\subsection{Action of the unitary group}

To investigate the invariance of the $h$-Dirac operators under the action of the unitary group $\widetilde{\mathrm{U}}(n)$ one needs the following result, see Lemma 11 in [25]. It turns out that the $h$-Dirac operators $\partial_{\underline{z}}$ and $\partial_{\underline{z}}^{\dagger}$ are the appropriate operators to work with in the Hermitian setting.

Lemma 1. Let $s \in \widetilde{U}(n)$ and consider the corresponding transformation $h(s)$ acting on the Clifford vector variable $\underline{X}$, i.e. $h(s): \underline{X} \longrightarrow s \underline{X} \bar{s}=s \underline{X} s^{\dagger}$. Then this transformation preserves the Hermitian decomposition of $\underline{X}$, i.e. $h(s)[\underline{z}]=s \underline{z} s^{\dagger} \in \mathbb{C} \Lambda_{n}$ and $h(s)\left[\underline{z}^{\dagger}\right]=s \underline{z}^{\dagger} s^{\dagger} \in \mathbb{C} \Lambda_{n}^{\dagger}$.

In order to recall that the $h$-representation of a spin group leads to associated operator actions on functions, one defines

$$
H(s)[g(\underline{X})]=s g(\bar{s} \underline{X} s) \bar{s}
$$

and

$$
L(s)[g(\underline{X})]=\operatorname{sg}(\bar{s} \underline{X} s) .
$$

As it is proved in [25], if $s \in \widetilde{\mathrm{U}}(n)$ then the $h$-Dirac operators $\partial_{\underline{z}}$ and $\partial_{\underline{z}}^{\dagger}$ commute with the operators $L(s)$ and $H(s)$, thus they are invariant under the unitary group action. Since the action of $H(s)$ on a $\mathbb{C}_{n}$-valued function $g$ may be converted into the action of $L(s)$ on $g I$, it is enough to study $L(s)$.

Theorem 1. For $s \in \widetilde{U}(n)$ one has

$$
\left[\partial_{\underline{z}}, L(s)\right]=0=\left[\partial_{\underline{z}}^{\dagger}, L(s)\right]
$$

where $[\cdot, \cdot]$ denotes the commutator of the two involved operators.

\subsection{The Cauchy-Kowalewskaya extension and the $h$-submonogenic system}

To study the Cauchy-Kowalewskaya problem for the $h$-monogenic system, it is most convenient to consider the system in $\mathbb{C}^{n+1}$ with variables $\left(z_{0}, z_{1}, \ldots, z_{n}\right)=\left(z_{0}, \underline{z}\right)$, i.e.:

$$
\left\{\begin{array}{c}
\left(\partial_{z_{0}} \mathfrak{f}_{0}^{\dagger}+\partial_{\underline{z}}\right) f=0 \\
\left(\partial_{z_{0}^{c}} \mathfrak{f}_{0}+\partial_{\underline{z}^{\dagger}}\right) f=0
\end{array}\right.
$$

where $\partial_{\underline{z}}=\sum_{j=1}^{n} \partial_{z_{j}} \mathfrak{f}_{j}^{\dagger}, \partial_{\underline{z}^{\dagger}}=\sum_{j=1}^{n} \partial_{z_{j}^{c}} \mathfrak{f}_{j}$ are the $h$-Dirac operators in $\mathbb{C}^{n}$. The CauchyKowalewskaya extension problem (in short CK-extension problem) arises naturally when looking to solutions in the form of a double power series

$$
f\left(z_{0}, z_{o}^{c}, \underline{z}, \underline{z}^{\dagger}\right)=\sum_{k, \ell=0}^{+\infty} z_{0}^{k}\left(z_{0}^{c}\right)^{\ell} f_{k \ell}\left(\underline{z}, \underline{z}^{\dagger}\right)
$$

and the solution is fully determined by its restriction to the boundary $k=0, \ell=0$. But the Cauchy data have to satisfy extra constraints in order to admit a CK-extension, and even the initial term $f_{00}$ has to. To obtain more clarity on the CK-extension problem first studied in $[16,35]$, in the paper [36] the authors considered a subsystem of the $h$-monogenic system that 
imposes no constraints on the initial term $f_{00}$. It is easily obtained as follows. Since one has that $\mathfrak{f}_{0} \mathfrak{f}_{0}^{\dagger}+\mathfrak{f}_{0}^{\dagger} \mathfrak{f}_{0}=1$ and

$$
\begin{aligned}
& \mathfrak{f}_{0} \mathfrak{f}_{0}^{\dagger}\left(\partial_{z_{0}} \mathfrak{f}_{0}^{\dagger}+\partial_{\underline{z}}\right)=\mathfrak{f}_{0} \mathfrak{f}_{0}^{\dagger} \partial_{\underline{z}} \\
& \mathfrak{f}_{0}^{\dagger} \mathfrak{f}_{0}\left(\partial_{z_{0}} \mathfrak{f}_{0}^{\dagger}+\partial_{\underline{z}}\right)=\partial_{z_{0}} \mathfrak{f}_{0}^{\dagger}+\mathfrak{f}_{0}^{\dagger} \mathfrak{f}_{0} \partial_{\underline{z}} \\
& \mathfrak{f}_{0}^{\dagger} \mathfrak{f}_{0}\left(\partial_{z_{0}^{c}} \mathfrak{f}_{0}^{\dagger}+\partial_{\underline{z}^{\dagger}}\right)=\mathfrak{f}_{0}^{\dagger} \mathfrak{f}_{0} \partial_{\underline{z}^{\dagger}} \\
& \mathfrak{f}_{0} \mathfrak{f}_{0}^{\dagger}\left(\partial_{z_{0}^{c}} \mathfrak{f}_{0}^{\dagger}+\partial_{\underline{z}^{\dagger}}\right)=\partial_{z_{0}^{c}} \mathfrak{f}_{0}+\mathfrak{f}_{0} \mathfrak{f}_{0}^{\dagger} \partial_{\underline{z}^{\dagger}}
\end{aligned}
$$

the $h$-monogenic system is clearly equivalent to what is called the $h$-submonogenic system in $[36]$

$$
\begin{aligned}
& \left(\partial_{z_{0}} \mathfrak{f}_{0}^{\dagger}+\mathfrak{f}_{0}^{\dagger} \mathfrak{f}_{0} \partial_{\underline{z}}\right) f=0 \\
& \left(\partial_{z_{0}} \mathfrak{f}_{0}+\mathfrak{f}_{0} \mathfrak{f}_{0}^{\dagger} \partial_{\underline{z}^{\dagger}}\right) f=0
\end{aligned}
$$

together with the constraints $\mathfrak{f}_{0} \mathfrak{f}_{0}^{\dagger} \partial_{\underline{z}} f=\mathfrak{f}_{0}^{\dagger} \mathfrak{f}_{0} \partial_{z^{\dagger}} f=0$. Since these constraints may be restricted to $k=0, \ell=0$ they are also satisfied by the Cauchy data (in fact by every term $f_{k \ell}$ in the series of $f$ ) and for the initial term $f_{00}$ these constraints are necessary and sufficient for the $h$ monogenic extension to exist.

To study the $h$-submonogenic system one has to write the solution into the form

$$
f=A+\mathfrak{f}_{0} B+\mathfrak{f}_{0}^{\dagger} C+\mathfrak{f}_{0} \mathfrak{f}_{0}^{\dagger} D
$$

where $A, B, C, D$ take values in the Clifford subalgebra with generators $\mathfrak{f}_{1}, \mathfrak{f}_{1}^{\dagger}, \ldots, \mathfrak{f}_{n}, \mathfrak{f}_{n}^{\dagger}$. The $h$-submonogenic system may be written in the form

$$
\begin{aligned}
& \partial_{z_{0}}(A+D)-\partial_{\underline{z}} C=0 \\
& \partial_{z_{0}} B+\partial_{\underline{z}} A=0 \\
& \partial_{z_{0}^{c}} B-\partial_{\underline{z}^{\dagger}} B=0 \\
& \partial_{z_{0}^{c}} C+\partial_{\underline{z}^{\dagger}}(A+D)=0
\end{aligned}
$$

where the elements $\mathfrak{f}_{0}, \mathfrak{f}_{0}^{\dagger}$ are being eliminated. To solve this system one again writes the solutions $A, B, C, D$ into the form of a double series solution $f=\sum_{k, \ell=0}^{+\infty} z_{o}^{k}\left(z_{0}^{c}\right)^{\ell} f_{k \ell}$ and the CK-extension problem may be solved by plugging these series into the system. The CK-extension problem discussed in [36] is quite involved but the initial term $A_{00}$ has no constraints. So $A_{00}$ may be any real analytic function and the CK-extension for the $h$-submonogenic system will be $h$-monogenic if and only if the initial conditions, and in particular the initial term $A_{00}$ satisfy the constraints $\mathfrak{f}_{0} \mathfrak{f}_{0}^{\dagger} \partial_{\underline{z}} f=\mathfrak{f}_{0}^{\dagger} \mathfrak{f}_{0} \partial_{\underline{z}^{\dagger}} f=0$. For the initial term $A_{00}$ these constraints are equivalent to the $h$-monogenic system $\partial_{\underline{z}} A_{00}=\partial_{\underline{z}^{\dagger}} A_{00}$ in one dimension less. In other words, the CK-extension problem as discussed in [16] is part of the CK-extension problem of the more general $h$-submonogenic system. In [36] several new special solutions of the $h$-submonogenic system were constructed, involving Bessel functions, hypergeometric functions and Laguerre polynomials; these were obtained by choosing the initial term $A_{00}$ in a special way and the obtained special solutions are $h$-submonogenic but not $h$-monogenic.

\section{Hermitian spherical monogenics and Fischer decomposition}

Definition 7. A polynomial $R_{k, l}\left(\underline{z}, \underline{z}^{\dagger}\right)$ is said to be homogeneous of degree $(k, l) \in \mathbb{N}^{2}$ if, for all $\eta \in \mathbb{C} \backslash\{0\}$,

$$
R_{k, l}\left(\eta \underline{z}, \eta^{c} \underline{\underline{\dagger}}^{\dagger}\right)=\eta^{k}\left(\eta^{c}\right)^{l} R_{k, l}\left(\underline{z}, \underline{z}^{\dagger}\right)
$$


An $h$-monogenic homogeneous polynomial $P_{k, l}\left(\underline{z}, \underline{z}^{\dagger}\right)$ of degree $(k, l)$ is called $h$-spherical monogenic of degree $(k, l)$.

Let us introduce the Euler and Gamma operators in the present setting. Define the Hermitian Gamma operators, or $h$-Gamma operators, as

$$
\Gamma_{z}=2 \underline{z} \wedge \partial_{\underline{z}}, \quad \Gamma_{z^{\dagger}}=2 \underline{z}^{\dagger} \wedge \partial_{\underline{z}}^{\dagger},
$$

and the Hermitian Euler operators, or $h$-Euler operators

$$
E_{\underline{z}}=\sum_{j=1}^{n} z_{j} \partial_{z_{j}}=\underline{z} \cdot \partial_{\underline{z}}, \quad E_{\underline{z}^{\dagger}}=\sum_{j=1}^{n} z_{j}^{c} \partial_{z_{j}^{c}}=\underline{z}^{\dagger} \cdot \partial_{\underline{z}}^{\dagger} .
$$

Recall that, in the affine setting, the traditional Euler and Gamma (or angular Dirac) operators are easily seen to commute, since the former is a purely radial operator, while the latter is angular. Although we will see that the angular character of the Gamma operator is not preserved in the present Hermitian setting, the commuting property remains.

Proposition 6. The $h$-Euler operators $E_{\underline{z}}, E_{\underline{z}^{\dagger}}$ commute with the $h$-Gamma operators $\Gamma_{\underline{z}}$, $\Gamma_{\underline{z}^{\dagger}}$.

Moreover the homogeneous polynomials are eigenfunctions of the $h$-Euler operators:

Proposition 7. Let $R_{k, l}\left(\underline{z}, \underline{z}^{\dagger}\right)$ be an homogeneous polynomial of degree $(k, l)$. Then

$$
E_{\underline{z}}\left[R_{k, l}\right]=k R_{k, l}, \quad E_{\underline{z}^{\dagger}}\left[R_{k, l}\right]=l R_{k, l} .
$$

Moreover, define a spherical monogenic of degree $(k, l)$ to be a homogeneous polynomial of degree $(k, l)$ which is $h$-monogenic; then

Proposition 8. Let $P_{k, l}$ be a spherical monogenic of degree $(k, l)$ then it is an eigenfunction of the $h$-Gamma operators, i.e.

$$
\Gamma_{\underline{z}}\left[R_{k, l}\right]=-k R_{k, l}, \quad \Gamma_{\underline{z}^{\dagger}}\left[R_{k, l}\right]=-l R_{k, l} .
$$

\subsection{The Fischer decomposition}

On the space $\mathcal{P}_{k, l}\left(\mathbb{C}_{2 n}\right)$ of all $\mathbb{C}_{2 n}$ valued homogeneous polynomials of degree $(k, l)$ one defines the so-called Fischer inner product as follows:

Definition 8. Let $R_{k, l}\left(\underline{z}, \underline{z}^{\dagger}\right)$ and $S_{k, l}\left(\underline{z}, \underline{z}^{\dagger}\right)$ be homogeneous polynomials of degree $(k, l)$. We define

$$
\left(R_{k, l} ; S_{k, l}\right)=\left[R_{k, l}^{\dagger}\left(2 \partial_{\underline{z}}^{\dagger}, 2 \partial_{\underline{z}}\right) S_{k, l}\left(\underline{z}, \underline{z}^{\dagger}\right)_{\mid \underline{z}=0}\right]_{0} .
$$

Note that in the above definition, it is in fact superfluous to take the restriction to $\underline{z}=0$ at the right hand side, since the action of the differential operator corresponding to $R_{k, l}^{\dagger}$ on the polynomial $S_{k, l}$ is a constant. However, this inner product may be extended to polynomials showing different degrees of homogeneity, where taking the restriction to $\underline{z}=0$ becomes necessary. The differential operator appearing in this definition originates from the given polynomial $R_{k, l}$ by Fischer duality. This Fischer duality is induced by the substitutions $\mathfrak{f}_{j} \rightarrow \mathfrak{f}_{j}^{\dagger}, \mathfrak{f}_{j}^{\dagger} \rightarrow \mathfrak{f}_{j}, i \rightarrow-i$, $x_{j} \rightarrow \partial_{x_{j}}, y_{j} \rightarrow \partial_{y_{j}}$, namely

$$
\underline{z}=\sum_{j=1}^{n} z_{j} \mathfrak{f}_{j}=\sum_{j=1}^{n}\left(x_{j}+i y_{j}\right) \mathfrak{f}_{j} \rightarrow \sum_{j=1}^{n}\left(\partial_{x_{j}}-i \partial_{y_{j}}\right) \mathfrak{f}_{j}^{\dagger}=2 \sum_{j=1}^{n} \partial_{z_{j}} \mathfrak{f}_{j}^{\dagger}=2 \partial_{\underline{z}}
$$


and

$$
\underline{z}^{\dagger}=\sum_{j=1}^{n} z_{j}^{c} \mathfrak{f}_{j}^{\dagger}=\sum_{j=1}^{n}\left(x_{j}-i y_{j}\right) \mathfrak{f}_{j}^{\dagger} \rightarrow \sum_{j=1}^{n}\left(\partial_{x_{j}}+i \partial_{y_{j}}\right) \mathfrak{f}_{j}=2 \sum_{j=1}^{n} \partial_{z_{j}^{c} \mathfrak{f}}=2 \partial_{\underline{z}}^{\dagger}
$$

such that an arbitrary polynomial $R_{k, l}\left(\underline{z}, \underline{z}^{\dagger}\right)$ indeed is converted into $R_{k, l}^{\dagger}\left(2 \partial_{\underline{z}}^{\dagger}, 2 \partial_{\underline{z}}\right)$. One may easily prove

Lemma 2. The Fischer inner product is a positive definite and Hermitian inner product on the space $\mathcal{P}_{k, l}\left(\mathbb{C}_{2 n}\right)$ of $\mathbb{C}_{2 n}$ valued homogeneous polynomials of degree $(k, l)$.

A first result is then obtained concerning the decomposition of an arbitrary homogeneous polynomial into a Hermitian spherical monogenic part and remaining terms.

Theorem 2 (Little Fischer decomposition). The space $\mathcal{P}_{k, l}$ of homogeneous polynomials of degree $(k, l)$ admits the orthogonal decomposition

$$
\mathcal{P}_{k, l}=\mathcal{M}_{k, l}^{+} \oplus\left\{\underline{z} \mathcal{P}_{k-1, l}+\underline{z}^{\dagger} \mathcal{P}_{k, l-1}\right\}
$$

Thus, for each homogeneous polynomial $R_{k, l}$ of degree $(k, l)$, there exists a unique $h$-spherical monogenic $P_{k, l}$ of degree $(k, l)$ and homogeneous polynomials $R_{k-1, l}^{(1)}$ and $R_{k, l-1}^{(2)}$, such that

$$
R_{k, l}=P_{k, l}+\left(\underline{z} R_{k-1, l}^{(1)}+\underline{z}^{\dagger} R_{k, l-1}^{(2)}\right)
$$

Note that, in the orthogonal decomposition above, the spherical monogenic $P_{k, l}$ is unique, since so is

$$
\widetilde{R}_{k, l}=\underline{z} R_{k-1, l}^{(1)}+\underline{z}^{\dagger} R_{k, l-1}^{(2)},
$$

however, the polynomials $R_{k-1, l}^{(1)}$ and $R_{k, l-1}^{(2)}$ are not uniquely determined, since $\underline{z} R_{k-1, l}^{(1)}+$ $\underline{z}^{\dagger} R_{k, l-1}^{(2)}=0$ does not imply that $R_{k-1, l}^{(1)}=R_{k, l-1}^{(2)}=0$.

Invoking the little Fischer decomposition, together with the above lemmata, one eventually arrives at the following important result.

Theorem 3 (Orthogonal Fischer decomposition).

(i) For each homogeneous polynomial of degree $(k, l) R_{k, l}$, there exist a unique spherical monogenic $P_{k, l}$, unique homogeneous polynomials $\underline{z} \widetilde{P}_{k-1, l}$ and $\underline{z}^{\dagger} \widetilde{P}_{k, l-1}$, and, for $p=$ $1,2, \ldots$, unique homogeneous polynomials $\underline{z}^{\dagger} P_{k-p, l-p}, \underline{z} \widetilde{P}_{k-p, l-p}, \underline{z}^{\dagger} P_{k-p, l-p-1}, \underline{z} \widetilde{P}_{k-p-1, l-p}$, such that

$$
\begin{aligned}
R_{k, l}=P_{k, l}+\underline{z} \widetilde{P}_{k-1, l}+\underline{z}^{\dagger} \widetilde{P}_{k, l-1}+\sum_{p}|\underline{z}|^{2 p-2}\left(\underline{z} \underline{z}^{\dagger} P_{k-p, l-p}+\underline{z} \underline{z}^{\dagger} \widetilde{P}_{k-p, l-p}\right) \\
+\sum_{p}|\underline{z}|^{2 p-2}\left(\underline{z}^{\dagger} \underline{z}^{\dagger} P_{k-p, l-p-1}+\underline{z} \underline{z}^{\dagger} \underline{z} \widetilde{P}_{k-p-1, l-p}\right)
\end{aligned}
$$

where $\widetilde{P}_{k-1, l}, \widetilde{P}_{k, l-1}, P_{k-p, l-p}, \widetilde{P}_{k-p, l-p}, P_{k-p, l-p-1}$ and finally $\widetilde{P}_{k-p-1, l-p}$ are spherical monogenics of the indicated degrees.

(ii) One has the following orthogonal decomposition of the space $\mathcal{P}_{k, l}$ of homogeneous polynomials of degree $(k, l)$ :

$$
\begin{aligned}
\mathcal{P}_{k, l}=\mathcal{M}_{k, l}^{+} & \oplus\left\{\underline{z} \mathcal{M}_{k-1, l}^{+}+\underline{z}^{\dagger} \mathcal{M}_{k, l-1}^{+}\right\} \\
& \oplus\left\{\underline{z z^{\dagger}} \mathcal{M}_{k-1, l-1}^{+}+\underline{z}^{\dagger} \underline{z} \mathcal{M}_{k-1, l-1}^{+}\right\} \\
& \oplus\left\{\underline{z z^{\dagger}} \underline{\mathcal{M}}_{k-2, l-1}^{+}+\underline{z}^{\dagger} \underline{z}^{\dagger} \mathcal{M}_{k-1, l-2}^{+}\right\} \\
& \oplus\left\{\underline{z z}^{\dagger} \underline{z z}^{\dagger} \mathcal{M}_{k-2, l-2}^{+}+\underline{z}^{\dagger} \underline{z z} \underline{\mathcal{M}}_{k-2, l-2}^{+}\right\} \oplus \ldots
\end{aligned}
$$


In [38] the author observes that, as in the classical case, the Fischer decomposition is completely determined by the Fischer decomposition of the reproducing kernel

$$
\mathcal{R}_{k, l}\left(\underline{z}, \underline{z}^{\dagger} ; \underline{u}, \underline{u}^{\dagger}\right)=\frac{\left\{\underline{z}^{\dagger}, \underline{u}\right\}\left\{\underline{z}, \underline{u}^{\dagger}\right\}}{k ! l !}
$$

in term of which any homogeneous polynomial $R_{k, l}$ can be written via the Fischer inner product. All the building blocks for $\mathcal{R}_{k, l}$ can all be expressed in terms of the spin Euler polynomials $P_{k}(\beta)$ and $P_{k}(n-\beta)$ with explicit formulae, see [38].

\section{Some integral formulae}

The material in this section is mainly taken from [13] to which the reader is referred for more information. In that paper the authors obtain the Cauchy and the Bochner-Martinelli formulae for $h$-monogenic functions using a matrix approach. First of all, let us introduce some useful differential forms:

$$
d \sigma_{\underline{z}}=\sum_{j=1}^{n}\left(\mathrm{f}_{j}^{\dagger}\left(d z_{1} \wedge d z_{1}^{c}\right) \wedge \ldots\left(\widehat{d z}_{j} \wedge d z_{j}^{c}\right) \wedge \ldots \wedge\left(d z_{n} \wedge d z_{n}^{c}\right)\right)
$$

and

$$
d \sigma_{\underline{z}^{\dagger}}=\sum_{j=1}^{n}\left(\mathfrak{f}_{j}\left(d z_{1} \wedge d z_{1}^{c}\right) \wedge \ldots\left(d z_{j} \wedge \widehat{d z_{j}^{c}}\right) \wedge \ldots \wedge\left(d z_{n} \wedge d z_{n}^{c}\right)\right)
$$

where ${ }^{\wedge}$ denotes that the element underneath is omitted, and the volume form

$$
d W\left(\underline{z}, \underline{z}^{\dagger}\right)=\left(d z_{1} \wedge d z_{1}^{c}\right) \wedge \ldots \wedge\left(d z_{n} \wedge d z_{n}^{c}\right) .
$$

The following result holds:

Theorem 4 (Hermitian Clifford-Stokes theorems). Let $\Omega$ be an open set in $\mathbb{R}^{2 n}$. Let $f, g$ be functions in $\mathcal{C}^{1}\left(\Omega, \mathbb{C}_{2 n}\right)$ and let $\Gamma$ be a $2 n$-dimensional differentiable, compact and oriented manifold with smooth boundary $\partial \Gamma$. Then

$$
\begin{gathered}
\int_{\partial \Gamma} f\left(\underline{z}, \underline{z}^{\dagger}\right) d \sigma_{\underline{z}} g\left(\underline{z}, \underline{z}^{\dagger}\right)=\int_{\Gamma}\left[\left(f \partial_{\underline{z}}\right) g+f\left(\partial_{\underline{z}} g\right)\right] d W\left(\underline{z}, \underline{z}^{\dagger}\right) \\
\int_{\partial \Gamma} f\left(\underline{z}, \underline{z}^{\dagger}\right)\left(-d \sigma_{\underline{z}^{\dagger}}\right) g\left(\underline{z}, \underline{z}^{\dagger}\right)=\int_{\Gamma}\left[\left(f \partial_{\underline{z}^{\dagger}}\right) g+f\left(\partial_{\underline{z}^{\dagger}} g\right)\right] d W\left(\underline{z}, \underline{z}^{\dagger}\right)
\end{gathered}
$$

Theorem 5 (Hermitian Cauchy theorems). Let $\Omega$ be an open set in $\mathbb{R}^{2 n}$. Let $g$ be a $h$ monogenic function in $\Omega$ and let $\Gamma$ be a $2 n$-dimensional differentiable, compact and oriented manifold with smooth boundary $\partial \Gamma$. Then

$$
\int_{\partial \Gamma} d \sigma_{\underline{z}} g\left(\underline{z}, \underline{z}^{\dagger}\right)=0 \quad \int_{\partial \Gamma} d \sigma_{\underline{z}^{\dagger}} g\left(\underline{z}, \underline{z}^{\dagger}\right)=0 .
$$

To prove the analogue of the Cauchy formula it is better to reformulate the notion of Hermitian monogenic functions by using suitable matrices (see [45]). To this end, one defines the set of the so-called circulant matrices of dimension $2 \times 2$, i.e. the set

$$
\mathscr{M}=\left\{\left[\begin{array}{ll}
m_{1} & m_{2} \\
m_{2} & m_{1}
\end{array}\right], \quad m_{1}, m_{2} \in \mathbb{C}_{2 n}\right\} .
$$


To each pair of continuously differentiable functions $g_{1}, g_{2}$ it is possible to associate the matrix

$$
\mathbf{G}=\left[\begin{array}{ll}
g_{1} & g_{2} \\
g_{2} & g_{1}
\end{array}\right]
$$

while to the pair of operators $\partial_{\underline{z}}, \partial_{\underline{z}^{\dagger}}$, one associates the matrix (see also [45]):

$$
\mathcal{D}=\left[\begin{array}{cc}
\partial_{\underline{z}} & \partial_{\underline{z}^{\dagger}} \\
\partial_{\underline{z}^{\dagger}} & \partial_{\underline{z}}
\end{array}\right]
$$

In particular, the following matrices will play a crucial role:

$$
\mathbf{E}=\frac{1}{(2 i)^{n}}\left[\begin{array}{cc}
\mathcal{E} & \mathcal{E}^{\dagger} \\
\mathcal{E}^{\dagger} & \mathcal{E}
\end{array}\right] \quad \mathbf{d} \boldsymbol{\Sigma}=\left[\begin{array}{cc}
d \sigma_{\underline{z}} & -d \sigma_{\underline{z}^{\dagger}} \\
-d \sigma_{\underline{z}^{\dagger}} & d \sigma_{\underline{z}}
\end{array}\right]
$$

where

$$
\mathcal{E}(\underline{z})=\frac{2}{a_{2 n}} \frac{\underline{z}}{|\underline{z}|^{2 n}}, \quad \mathcal{E}^{\dagger}(\underline{z})=\frac{2}{a_{2 n}} \frac{\underline{z}^{\dagger}}{|\underline{z}|^{2 n}},
$$

and $a_{2 n}$ is the area of the unit sphere in $\mathbb{R}^{2 n}$. Note that $\mathcal{E}, \mathcal{E}^{\dagger}$ are not the fundamental solutions for the operator $\partial_{\underline{z}}$ or $\partial_{\underline{z}^{\dagger}}$.

Definition 9. We say that the matrix of functions $\mathbf{G}$ defined on $\Omega$ is left Hermitian monogenic, or $H$-monogenic for short, on $\Omega$ if and only if

$$
\mathcal{D} \mathbf{G}=\mathbf{O},
$$

where $\mathbf{O}$ denote the zero $2 \times 2$ matrix.

Remark 8. It is easy to verify that matrices of the form

$$
\left[\begin{array}{ll}
g & 0 \\
0 & g
\end{array}\right]
$$

are $H$-monogenic if and only if $g$ is $h$-monogenic. Thus any $H$-monogenic matrix of the above form can be identified with its element $g$, and conversely any $h$-monogenic function $g$ can be identified with a $H$-monogenic matrix of the above form.

To state the analogue of the Cauchy formula some more notations are needed: let $\underline{Y}$ be a vector in $\mathbb{R}^{2 n}$ and let $\underline{Y} \mid$ be its twist; denote by $\underline{v}, \underline{v}^{\dagger}$ the corresponding $h$-variables

$$
\underline{v}=\frac{1}{2}(\underline{Y}+i \underline{Y} \mid) \quad \underline{v}^{\dagger}=-\frac{1}{2}(\underline{Y}-i \underline{Y} \mid) .
$$

Theorem 6 (Cauchy formula). Let $\Omega$ be an open set in $\mathbb{R}^{2 n}, g_{1}, g_{2} \in \mathcal{C}^{1}\left(\Omega, \mathbb{C}_{2 n}\right)$ and let $\mathbf{G}$ be the corresponding matrix as in (5.3). Let $\Gamma \subset \Omega$ be a $2 n$-dimensional compact, differentiable, oriented manifold with smooth boundary $\partial \Gamma$ and let $\underline{Y}$ in the interior of $\Gamma$. If $\mathbf{G}$ is $H$ monogenic in $\Omega$ then

$$
\mathbf{G}\left(\underline{v}, \underline{v}^{\dagger}\right)=\int_{\partial \Gamma} \mathbf{E}(\underline{z}-\underline{v}) \mathbf{d} \boldsymbol{\Sigma}_{\left(\underline{z}, \underline{z}^{\dagger}\right)} \mathbf{G}\left(\underline{z}, \underline{z}^{\dagger}\right) .
$$

Remark 9. The theorem holds in particular when $\mathbf{G}$ is of the form (5.4). Let us now restrict the attention to functions taking values in the complex spinor space $\mathbb{C S}=\mathbb{C}_{2 n} I \cong \mathbb{C}_{n} I$. Let us set $\mathbb{C S}_{j}=\left(\mathbb{C} \Lambda_{n}^{\dagger}\right)^{(j)} I$ for $j=1, \ldots, n$ so that $\mathbb{C S}$ decomposes as $\mathbb{C S}=\oplus_{j=1}^{n} \mathbb{C S}_{j}$ and let $g$ be a $\mathbb{C S}_{n}$-valued function. Since $\mathbb{C S}_{n}$ is generated by $\mathfrak{f}_{1} \ldots \mathfrak{f}_{n} I$, a $\mathbb{C S}_{n}$-valued function has the form $g\left(z_{1}, \ldots, z_{n}\right)=g_{n}\left(z_{1}, \ldots, z_{n}\right) \mathfrak{f}_{1} \ldots \mathfrak{f}_{n} I$ where $g_{n}$ is a smooth function. The fact that $g$ is $h$-monogenic translates into the fact that $g_{n}$ is holomorphic in $z_{1}, \ldots, z_{n}$, see proposition 5. The Cauchy integral formula gives precisely the well known Bochner-Martinelli formula. 


\section{Hermitian Radial Algebra}

To define the so-called Hermitian radial algebra one starts from a basic set $S$ of abstract complex vector variables $Z, U, \ldots$, which are merely symbols. As multiplication rules, we assume that

$$
\left(A_{1}\right) \quad Z U=-U Z, \quad Z^{2}=0, \quad Z, U \in S
$$

and we hence know that the associative algebra $\operatorname{Alg}(S)$ generated by $S$ over the field of complex numbers is a generalized Grassmann algebra (a usual Grassmann algebra in case $S$ is finite). Then, consider another set $S^{\dagger}$ of symbols, disjoint from $S$, which is in one-to-one correspondence with $S$, i.e. there exists a bijective map

$$
\dagger: S \rightarrow S^{\dagger}
$$

One also assumes the axiom

$$
\left(A_{1}^{\dagger}\right) \quad Z^{\dagger} U^{\dagger}=-U^{\dagger} Z^{\dagger}, \quad Z^{\dagger^{2}}=0, Z, U \in S
$$

so that $\operatorname{Alg}\left(S^{\dagger}\right)$ is a copy of $\operatorname{Alg}(S)$ and also one assumes that $Z U^{\dagger}+U^{\dagger} Z$ is scalar for all $Z \in S, U^{\dagger} \in S^{\dagger}$ which is equivalent to assume the identity

$$
\left(A_{2}\right) \quad\left[V,\left\{Z, U^{\dagger}\right\}\right]=0, \quad\left[V^{\dagger},\left\{Z, U^{\dagger}\right\}\right]=0, \quad V, Z \in S, U^{\dagger}, V^{\dagger} \in S^{\dagger} .
$$

Under these assumptions the complete associative algebra $R(S, \dagger)$, generated by the whole set $S \cup S^{\dagger}$ with the previous axioms is a "radial algebra" in the sense of [47]. With the notation

$$
(U, Z)=\left(Z^{\dagger}, U^{\dagger}\right)=\left\{U^{\dagger}, Z\right\}
$$

it follows that

$$
\begin{gathered}
|Z|^{2}=(Z, Z)=\left(Z^{\dagger}, Z^{\dagger}\right)=\left|Z^{\dagger}\right|^{2} \\
\left(U, Z^{\dagger}\right)=\left(Z^{\dagger}, U\right)=0 .
\end{gathered}
$$

together with the constraints

$$
\left(C_{1}\right) \quad|Z|^{2} \quad \text { is real valued }
$$

$\left(C_{2}\right) \quad(U, Z) \quad$ is the complex conjugate of $(Z, U)$.

The radial algebra $R(S, \dagger)$ can be represented in terms of a Clifford algebra. To that end, choose a dimension $m$ and introduce the Clifford algebra $\mathbb{C}_{2 n}$ with a Witt basis $\mathfrak{f}_{1}, \ldots, \mathfrak{f}_{n}$; $\mathfrak{f}_{1}^{\dagger}, \ldots, \mathfrak{f}_{n}^{\dagger}$. For any variable $Z \in S$, select a $t$-uple of complex variables $\left(X_{1}, \ldots, X_{n} ; Y_{1}, \ldots, Y_{n}\right)$ with no intersection for two different variables $Z, U \in S$. Then the Witt basis together with the collection of all the complex variables taken together generate the Clifford polynomial algebra $P(n, S)$ determined by the dimension $n$ and the set of labels $S$. Then consider a representation

$$
\stackrel{-}{ }: R(S, \dagger) \rightarrow P(n, S)
$$

which is determined by

$$
Z \rightarrow \underline{Z}=\sum \mathfrak{f}_{j} Z_{j}, \quad Z^{\dagger} \rightarrow \underline{Z}^{\dagger}=\sum \mathfrak{f}_{j}^{\dagger} Z_{j}^{\dagger}
$$

with $Z_{j}=X_{j}+i Y_{j}, Z_{j}^{\dagger}=X_{j}-i Y_{j}$. Then, following [47], it is possible to prove the following 
Theorem 7. In case $S$ is finite with cardinality $\ell$; then for any $n \geq \ell$ the representation $\therefore: R(S, \dagger) \rightarrow P(n, S)$ is an isomorphism.

The representation " - " hence leads to the possibility to replace abstract vector variables like $Z, U, Z^{\dagger}, U^{\dagger}, \ldots$ by Clifford vector variables $\underline{Z}, \underline{U}, \underline{Z}^{\dagger}, \underline{U}^{\dagger}, \ldots$, so that in order to verify whether a certain identity holds on the abstract level (i.e. follows from the axioms) it suffices to verify the property in a Clifford algebra of sufficiently high dimension. The Hermitian vector derivatives $\partial_{Z}$ and $\partial_{Z}^{\dagger}$ are introduces as follows. They can be defined as endomorphisms on the algebra $R(S, \dagger)$. Both operators $\partial_{Z}, \partial_{Z}^{\dagger}$ can act from both sides on elements $F \in R(S, \dagger)$. Small letters $f$ are used to denote scalar valued elements i.e. elements belonging to the center $Z R(S, \dagger)$, which is generated by the inner products $\{v, w\}$, for $v, w \in S \cup S^{\dagger}$. The axioms to define $\partial_{Z}, \partial_{Z}^{\dagger}$ are similar to those used to define $\partial_{x}$ in [47]:

$$
\begin{gathered}
\left(D_{1}\right) \quad \partial_{Z}[f F]=\partial_{Z}[f] F+f \partial_{Z}[F], \quad \partial_{Z}^{\dagger}[f F]=\partial_{Z}^{\dagger}[f] F+f \partial_{Z}^{\dagger}[F] \\
{[f F] \partial_{Z}=F \partial_{Z}[f]+f[F] \partial_{Z}, \quad[f F] \partial_{Z}^{\dagger}=F \partial_{Z}^{\dagger}[f]+f[F] \partial_{Z}^{\dagger}} \\
\left(D_{2}\right) \quad \partial_{Z}[G]=[G] \partial_{Z}=0 \quad \text { for } \quad G \in \operatorname{Alg}\left((S \backslash Z) \cup S^{\dagger}\right) \\
\partial_{Z}^{\dagger}[G]=[G] \partial_{Z}^{\dagger}=0 \quad \text { for } \quad G \in \operatorname{Alg}\left(S \cup\left(S^{\dagger} \backslash Z^{\dagger}\right)\right)
\end{gathered}
$$

These are the basic derivation axioms which allow one to reduce the problem of evaluation of $\partial_{Z}[F]$ (resp. $[F] \partial_{Z}, \partial_{Z}^{\dagger}[F],[F] \partial_{Z}^{\dagger}$ ) to the evaluation of scalar valued objects and the objects $\partial_{Z}[Z],[Z] \partial_{Z}, \partial_{Z}^{\dagger}\left[Z^{\dagger}\right],\left[Z^{\dagger}\right] \partial_{Z}^{\dagger}$. Indeed, any $F \in R(S, \dagger)$ consists of a sum of products of elements $v_{1} \ldots v_{t}, v_{j} \in S \cup S^{\dagger}$. In case $Z$ is not among the vectors $v_{j}$ then by $\left(D_{2}\right)$ the evaluation vanishes and otherwise one can bring all the $Z$ 's in front using the commutation relations

$$
U Z=-Z U, \quad U^{\dagger} Z=-Z U^{\dagger}+\left\{U^{\dagger}, Z\right\}
$$

This means that one only has to be able to evaluate objects of the form $f G$ with $f$ scalar and $Z$ not occurring in $G$ or of the form $f Z G$ with $f$ scalar and $Z$ not occurring in $G$. Now, as to the evaluation of scalars, assume

$$
\left(D_{3}\right) \quad \partial_{Z}(U, Z)=U^{\dagger}, \quad \partial_{Z}^{\dagger}(Z, U)=\partial_{Z}^{\dagger}\left(U^{\dagger}, Z^{\dagger}\right)=U
$$

and this for every $Z \in S, U \in S$ (including $Z=U$ ). Also assume

$$
\left(D_{4}\right) \quad \partial_{Z}[Z]=\frac{1}{2}(n+B), \quad \partial_{Z}^{\dagger}\left[Z^{\dagger}\right]=\frac{1}{2}(n-B)
$$

and

$$
\left(D_{4}^{\prime}\right) \quad[Z] \partial_{Z}=\frac{1}{2}(n-B), \quad\left[Z^{\dagger}\right] \partial_{Z}^{\dagger}=\frac{1}{2}(n+B)
$$

whereby $n$ is scalar and $B$ is a bivector. The universality of $n$ and $B$ follows from the obvious assumption

$$
(U) \quad \partial_{Z}[Z]=\partial_{U}[U], \quad \partial_{Z}^{\dagger}\left[Z^{\dagger}\right]=\partial_{U}^{\dagger}\left[U^{\dagger}\right] .
$$

To define a bivector $B$ as above one sets

$$
\left(D_{5}\right) \quad B=\partial_{Z}[Z]-\partial_{Z}^{\dagger}\left[Z^{\dagger}\right]
$$

and from the Clifford representation one has relations of the form

$$
\left(A_{3}\right) \quad B Z-Z B=-2 Z, \quad B Z^{\dagger}-Z^{\dagger} B=2 Z .
$$


This axiom together with $\left(A_{1}\right),\left(A_{2}\right)$ determines the associative algebra $R(S, \dagger, n, B)$ generated over the field of rational functions in $n$ by the set $S \cup S^{\dagger} \cup B$. Moreover one can verify that for any vectorial object $v$ in the algebra, $v B-B v$ is still a vector. One extends (D2) by requiring

$$
\left(D_{2}\right) \quad \partial_{Z}[B]=\partial_{Z^{\dagger}}[B]=[B] \partial_{Z}=[B] \partial_{Z^{\dagger}}=0, \quad \partial_{Z}[n]=\partial_{Z^{\dagger}}[n]=0 .
$$

From the Clifford setting it also follows that

$$
B \partial_{Z^{\dagger}}-\partial_{Z^{\dagger}} B=-2 \partial_{Z^{\dagger}}, \quad B \partial_{Z}-\partial_{Z} B=2 \partial_{Z}
$$

but this is no axiom; it can be derived from the abstract setting as well. Hence to define the Hermitian radial algebra one needs

1. the axioms of radial algebra $\left(A_{1}\right),\left(A_{2}\right)$ and $\left(A_{3}\right)$ including the scalar $n$

2. the derivation axioms $\left(D_{1}\right),\left(D_{2}\right)$

3. the evaluation of scalars $\left(D_{3}\right)$

4. the axioms $\left(D_{4}\right),\left(D_{4}^{\prime}\right)$ together with the obvious axiom $(U)$.

\section{Complexes of Hermitian Dirac operators}

This section describes the algebraic analysis of the $h$-hermitian system. It is interesting to note that the various notions of hyperholomorphy, in one variable, are described by systems that are associated, in the sense that will be made precise below, to square matrices and in this sense they are not interesting. The system arising from the condition of $h$-monogenicity is not square and gives rise to an interesting analysis. In order to keep this note self-contained, some basic notions in algebraic analysis are repeated here but the reader is referred to [28] for more information. Let $\vec{f}=\left(f_{1}, \ldots, f_{r}\right)$ be an $r$-tuple of functions real differentiable on an open set $U \subseteq \mathbb{R}^{m}$ and let

$$
\sum_{j=1}^{r} P_{i j}(D) f_{j}=g_{i} \quad i=1, \ldots, q
$$

be a $q \times r$ system of linear partial differential equations with constant coefficients. Let $P=\left[P_{i j}\right]$ be an $q \times r$ matrix of complex polynomials in $\mathbb{C}^{m}$ and $D=\left(-i \partial_{x_{1}}, \ldots,-i \partial_{x_{m}}\right)$. The matrix $P$, symbol of the system, can be obtained from $P(D)=\left[P_{i j}(D)\right]$ by replacing (formally) $\partial_{x_{k}}$ by the complex variable $z_{k}$ for every $k=1, \ldots, m$. Let $R$ be the ring of polynomials with complex coefficients $\mathbb{C}\left[z_{1}, \ldots, z_{m}\right]$. The transpose matrix $P^{t}$ of $P$ is an $R$-homomorphism $R^{q} \rightarrow R^{r}$ whose cokernel is $R^{r} / P^{t} R^{q}=R^{r} /\left\langle P^{t}\right\rangle$ is denoted by $M$. Note that $\left\langle P^{t}\right\rangle$ is the submodule of $R^{r}$ generated by the columns of $P^{t}$. The Hilbert syzygy theorem guarantees the existence of a finite free resolution of the form

$$
0 \longrightarrow R^{a_{s}} \stackrel{P_{a_{s}}^{t}}{\longrightarrow} R^{a_{s-1}} \longrightarrow \ldots \stackrel{P_{1}^{t}}{\longrightarrow} R^{q} \stackrel{P^{t}}{\longrightarrow} R^{r} \longrightarrow M \longrightarrow 0
$$

that together with its transpose

$$
0 \longrightarrow R^{r} \stackrel{P}{\longrightarrow} R^{q} \stackrel{P_{1}}{\longrightarrow} \ldots \longrightarrow R^{a_{s-1}} \stackrel{P_{a_{s}}}{\longrightarrow} R^{a_{s}} \longrightarrow 0
$$

are key tools for the algebraic analysis of the given system. The matrix $P_{a_{i}}^{t}(D)$ gives the compatibility conditions for the system whose symbol is the polynomial matrix $P_{a_{i-1}}^{t}$. In particular, 
$P_{1}(D)$ gives the compatibility conditions that a datum $\vec{g}$ of a inhomogeneous system $P(D) \vec{f}=\vec{g}$ must satisfy to have solutions $\vec{f}$. The cohomology groups of $(7.6)$, denoted by $\operatorname{Ext}^{j}(M, R)$ are uniquely determined by $M$ (even though (7.6) is not uniquely defined) and carry analytic information on the nullsolutions to the system associated to $P(D)$. For example, the vanishing of $\operatorname{Ext}^{1}(M, R)$ is related to the removability of compact singularities.

Our first goal is now to write the matrix associated to the $h$-hermitian system. To this end, one needs the following result:

Theorem 8. For all $1 \leq j \leq n-1$, the spaces $\mathbb{C S}_{j}$ are $\mathfrak{s l}(n)$-irreducibles with highest weight $\left(1_{n-j}, 0_{j-1}\right)$, under the multiplicative $\mathfrak{s l}(n)$-action. Both $\mathbb{C S}_{0}$ and $\mathbb{C S}_{n}$ yield a copy of the trivial representation.

Note that, whereas $\underline{X}$ and $\partial_{\underline{X}}$ define endomorphisms of $\mathbb{C S}$ under the embedding $\mathbb{C}^{2 n} \hookrightarrow$ $\mathbb{C}_{2 n}^{(1)}$, the complex vector variables $\left(\underline{z}, \underline{z}^{\dagger}\right)$ and Hermitian Dirac operators $\left(\partial_{\underline{z}}, \partial_{\underline{z}}^{\dagger}\right)$ map spaces $\mathbb{C S}_{j}$ into $\mathbb{C S}_{j \pm 1}$, where $\mathbb{C S}_{-1}=\mathbb{C S}_{n+1}=0$. This fact allows to write the matrices associated to the operators $\partial_{\underline{z}}, \partial_{\underline{z}}^{\dagger}$ in a special form. With an abuse, the matrices are denoted with the same symbol as the operators.

Proposition 9. With respect to the decomposition $\mathbb{C S}=\oplus_{j=0}^{n} \mathbb{C S}_{j}$ we can represent the Dirac operators as the following block matrices with entries in $\mathbb{C}\left[\underline{z}, \underline{z}^{\dagger}\right]$

$$
\partial_{\underline{z}}=\left[\begin{array}{ccccc}
0 & 0 & \cdots & 0 & 0 \\
d_{1} & 0 & \cdots & 0 & 0 \\
0 & d_{2} & \ddots & 0 & 0 \\
\vdots & \vdots & \ddots & \ddots & \vdots \\
0 & 0 & \cdots & d_{n} & 0
\end{array}\right]
$$

where the $i, j$-th block is a $\left(\begin{array}{l}n \\ i\end{array}\right)$ times $\left(\begin{array}{l}n \\ j\end{array}\right)$ matrix of either zeroes or given by the restriction of the Dirac operator $d_{i}:=\pi_{\mathbb{C S}_{i}} \circ\left[\partial_{\underline{z}}\right]_{\mathbb{C S}_{i-1}}: \mathbb{C S}_{i-1} \rightarrow \mathbb{C S}_{i}$, and similarly

$$
\partial_{\underline{z}}^{\dagger}=\left[\begin{array}{ccccc}
0 & \delta_{0} & 0 & \cdots & 0 \\
0 & 0 & \delta_{1} & \cdots & 0 \\
\vdots & \vdots & \ddots & \ddots & \vdots \\
0 & 0 & 0 & \ddots & \delta_{n-1} \\
0 & 0 & 0 & \cdots & 0
\end{array}\right]
$$

where $\delta_{i}:=\pi_{\mathbb{C S}_{i}} \circ\left[\partial_{\underline{z}}^{\dagger}\right]_{\mathbb{C S}_{i+1}}: \mathbb{C S}_{i+1} \rightarrow \mathbb{C S}_{i}$.

Remark 10. It can be verified by direct computations that the operators $d_{i}$ and $\delta_{i}$ satisfy $d_{i} d_{i-1}=\delta_{i} \delta_{i+1}=0$, for all $i$ such that the equalities make sense.

Next result describes more precisely the resolution of the module associated to the Hermitian Dirac system and the maps associated to the first syzygies.

Theorem 9. The free resolution of the module associated to the Hermitian Dirac system in dimension $2 n$ is linear of length $n$. The first syzygies are described by the relations

$$
\left\{\begin{array}{lll}
d_{i} a_{\mid \mathbb{C S}_{i-1}}=0 & & 2 \leq i \leq n, \\
\delta_{i} b_{\mid \mathbb{C S}_{i+1}}=0 & & 0 \leq i \leq n-2,
\end{array}\right.
$$

which form $2^{n}-n-1$ complex relations among the scalar components of $a$ and $b$. 
Remark 11. It is interesting to note that, unlike what happens for the Cauchy-Riemann or the Cauchy-Fueter system, the compatibility conditions do not contain relations involving both $a$ and $b$ at a time.

The proof of the previous theorem gives an explicit description of the whole resolution for the module $M$. This fact i s, in general, non trivial (see [28]). In this case, the particular form of the matrix associated to the Hermitian system allows to write the maps in the sequence.

Theorem 10. Let $\varphi_{0}=P^{t}$ and $\varphi_{j}$ be the $j$-th maps in the minimal free resolution of the module $M=R^{2^{n}} / \operatorname{Im}\left(P^{t}\right)$. Its nonzero blocks are only the matrices associated to $d_{j+1}, \ldots, d_{n}$ and $\delta_{0}, \ldots, \delta_{n-j-1}$. Explicitly, the matrix $\varphi_{j}$ for $j \geq 1$ is given by the transpose of

$$
\left[\begin{array}{cccccccc}
d_{j+1} & 0 & 0 & 0 & 0 & 0 & 0 & 0 \\
0 & \ddots & 0 & 0 & 0 & 0 & 0 & 0 \\
0 & 0 & d_{n} & 0 & 0 & 0 & 0 & 0 \\
0 & 0 & 0 & 0 & 0 & \delta_{0} & 0 & 0 \\
0 & 0 & 0 & 0 & 0 & 0 & \ddots & 0 \\
0 & 0 & 0 & 0 & 0 & 0 & 0 & \delta_{n-j-1}
\end{array}\right]
$$

The Betti numbers of $M$ are given by

$$
\beta_{0}=2^{n}, \text { and } \quad \beta_{i}=2\left(\sum_{j=i}^{n}\left(\begin{array}{l}
n \\
j
\end{array}\right)\right), \quad 1 \leq i \leq n .
$$

As a consquence of this discussion, one may obtain important information on $h$-monogenic functions. For example, one deduces the Hartogs' type of phenomenon does not hold:

Corollary 1. Let $K$ be a compact convex subset of an open set $U \subseteq \mathbb{R}^{2 n}, n>1$ and let $f$ be a Hermitian monogenic function on $U \backslash K$. Then $f$ cannot in general be extended to a Hermitian monogenic function on $U$.

The case of functions $h$-hermitian in several variables has been treated in [32].

\section{References}

[1] R. Abreu-Blaya, J. Bory-Reyes, F. Brackx, H. De Schepper, and F. Sommen. A Hermitian Cauchy formula on a domain with fractal boundary. J. Math. Anal. Appl., 369(1):273-282, 2010.

[2] R. Abreu-Blaya, J. Bory-Reyes, F. Brackx, H. De Schepper, and F. Sommen. A Hilbert transform for matrix functions on fractal domains. Complex Anal. Oper. Theory, 6(2):359$372,2012$.

[3] Ricardo Abreu Blaya, Juan Bory Reyes, Fred Brackx, Bram De Knock, Hennie De Schepper, Dixan Peña Peña, and Frank Sommen. Hermitean Cauchy integral decomposition of continuous functions on hypersurfaces. Bound. Value Probl., pages Art. ID 425256, 16, 2008 .

[4] Ricardo Abreu-Blaya, Juan Bory-Reyes, Fred Brackx, Hennie De Schepper, and Frank Sommen. Boundary value problems associated to a Hermitian Helmholtz equation. $J$. Math. Anal. Appl., 389(2):1268-1279, 2012. 
[5] Ricardo Abreu-Blaya, Juan Bory-Reyes, Fred Brackx, Hennie De Schepper, and Frank Sommen. Cauchy integral formulae in quaternionic Hermitean Clifford analysis. Complex Anal. Oper. Theory, 6(5):971-985, 2012.

[6] Ricardo Abreu Blaya, Juan Bory Reyes, and Tania Moreno García. Hermitian decomposition of continuous functions on a fractal surface. Bull. Braz. Math. Soc. (N.S.), 40(1):107$115,2009$.

[7] R. Abreu-Blaya, J. Bory-Reyes, R. Delanghe, F. Sommen, Duality for Hermitean systems in $\mathbb{R}^{2 n}$, Compl. Anal. Oper. Theory. 1: 341-357, 2012.

[8] Fred Brackx, Jarolím Bureš, Hennie De Schepper, David Eelbode, Frank Sommen, and Vladímir Souček. Fundaments of Hermitean Clifford analysis. I. Complex structure. Complex Anal. Oper. Theory, 1(3):341-365, 2007.

[9] F. Brackx, J. Bureš, H. De Schepper, D. Eelbode, F. Sommen, and V. Souček. Fundaments of Hermitean Clifford analysis. II. Splitting of $h$-monogenic equations. Complex Var. Elliptic Equ., 52(10-11):1063-1079, 2007.

[10] F. Brackx, B. De Knock, and H. De Schepper. A matrix Hilbert transform in Hermitean Clifford analysis. J. Math. Anal. Appl., 344(2):1068-1078, 2008.

[11] F. Brackx, B. De Knock, and H. De Schepper. The Hermitean Hilbert-Dirac connection. Adv. Appl. Clifford Algebr., 19(2):211-224, 2009.

[12] F. Brackx, B. De Knock, H. De Schepper, N. De Schepper, and F. Sommen. A new Hilbert transform in Hermitean Clifford analysis. In Function spaces in complex and Clifford analysis, pages 109-126. Natl. Univ. Publ. Hanoi, Hanoi, 2008.

[13] F. Brackx, B. De Knock, H. De Schepper, and F. Sommen. On Cauchy and MartinelliBochner integral formulae in Hermitean Clifford analysis. Bull. Braz. Math. Soc. (N.S.), 40(3):395-416, 2009.

[14] F. Brackx, H. De Schepper, N. De Schepper, and F. Sommen. Hermitean Clifford-Hermite polynomials. Adv. Appl. Clifford Algebr., 17(3):311-330, 2007.

[15] F. Brackx, H. De Schepper, N. De Schepper, and F. Sommen. Hermitian Clifford-Hermite wavelets: an alternative approach. Bull. Belg. Math. Soc. Simon Stevin, 15(1):87-107, 2008.

[16] F. Brackx, H. De Schepper, R. Lávička, and V. Souček. The Cauchy-Kovalevskaya extension theorem in Hermitian Clifford analysis. J. Math. Anal. Appl., 381(2):649-660, 2011.

[17] F. Brackx, H. De Schepper, R. Lávička, and V. Souček. Gel' fand-Tsetlin bases of orthogonal polynomials in Hermitean Clifford analysis. Math. Methods Appl. Sci., 34(17):2167-2180, 2011.

[18] F. Brackx, H. De Schepper, M. E. Luna-Elizarrarás, and M. Shapiro. On fundamental solutions in Clifford analysis. Complex Anal. Oper. Theory, 6(2):325-339, 2012.

[19] F. Brackx, H. De Schepper, M. E. Luna-Elizarrarás, and M. Shapiro. The Teodorescu operator in Clifford analysis. Chin. Ann. Math. Ser. B, 33(4):625-640, 2012.

[20] F. Brackx, H. De Schepper, and V. Souček. Differential forms versus multi-vector functions in Hermitean Clifford analysis. Cubo, 13(2):85-117, 2011. 
[21] Fred Brackx, Hennie De Schepper, Nele De Schepper, David Eelbode, and Frank Sommen. Orthogonality of the generalized Hermitean Clifford-Hermite polynomials. Integral Transforms Spec. Funct., 19(9-10):687-707, 2008.

[22] Fred Brackx, Hennie De Schepper, Nele De Schepper, and Frank Sommen. Generalized Hermitean Clifford-Hermite polynomials and the associated wavelet transform. Math. Methods Appl. Sci., 32(5):606-630, 2009.

[23] Fred Brackx, Hennie De Schepper, David Eelbode, and Vladimir Souček. The Howe dual pair in Hermitean Clifford analysis. Rev. Mat. Iberoam., 26(2):449-479, 2010.

[24] Fred Brackx, Hennie De Schepper, and Frank Sommen. A theoretical framework for wavelet analysis in a Hermitean Clifford setting. Commun. Pure Appl. Anal., 6(3):549-567, 2007.

[25] Fred Brackx, Hennie De Schepper, and Frank Sommen. The Hermitian Clifford analysis toolbox. Adv. Appl. Clifford Algebr., 18(3-4):451-487, 2008.

[26] Fred Brackx, Hennie De Schepper, and Vladimír Souček. Fischer decompositions in Euclidean and Hermitean Clifford analysis. Arch. Math. (Brno), 46(5):301-321, 2010.

[27] F. Brackx, R. Delanghe, F. Sommen, Clifford Analysis, Pitman Publishers (BostonLondon-Melbourne, 1982).

[28] Fabrizio Colombo, Irene Sabadini, Franciscus Sommen, and Daniele C. Struppa. Analysis of Dirac systems and computational algebra, volume 39 of Progress in Mathematical Physics. Birkhäuser Boston Inc., Boston, MA, 2004.

[29] Fabrizio Colombo, Irene Sabadini, and Daniele C. Struppa. Duality theorems in the Hermitian setting. J. Math. Anal. Appl., 390(1):47-58, 2012.

[30] Alberto Damiano and David Eelbode. Invariant operators between spaces of $h$-monogenic polynomials. Adv. Appl. Clifford Algebr., 19(2):237-251, 2009.

[31] Alberto Damiano and David Eelbode. Hermitian generalization of the Rarita-Schwinger operators. Acta Math. Sin. (Engl. Ser.), 26(2):311-330, 2010.

[32] Alberto Damiano, David Eelbode, and Irene Sabadini. Algebraic analysis of Hermitian monogenic functions. C. R. Math. Acad. Sci. Paris, 346(3-4):139-142, 2008.

[33] Alberto Damiano, David Eelbode, and Irene Sabadini. Invariant syzygies for the Hermitian Dirac operator. Math. Z., 262(4):929-945, 2009.

[34] Alberto Damiano, David Eelbode, and Irene Sabadini. Quaternionic Hermitian spinor systems and compatibility conditions. Adv. Geom., 11(1):169-189, 2011.

[35] Bram De Knock, Dixan Peña Peña, and Frank Sommen. On the CK-extension for a special overdetermined system in complex Clifford analysis. In Hypercomplex analysis, Trends Math., pages 115-124. Birkhäuser Verlag, Basel, 2009.

[36] Nele De Schepper, Dixan Peña Peña, and Frank Sommen. Special functions and systems in Hermitian Clifford analysis. Math. Methods Appl. Sci. 36(11): 1471-1484, 2013.

[37] Richard Delanghe, Franciscus Sommen, Vladimir Soucek. Clifford algebra and spinor-valued functions. A function theory for the Dirac operator. Kluwer Academic Publishers Group, Dordrecht, 1992. 
[38] D. Eelbode. Stirling numbers and spin-Euler polynomials. Experiment. Math., 16(1):55-66, 2007.

[39] David Eelbode. A Clifford algebraic framework for $\mathfrak{s p}(m)$-invariant differential operators. Adv. Appl. Clifford Algebr., 17(4):635-649, 2007.

[40] David Eelbode. Zonal Hermitian monogenic functions. In Complex analysis and its applications, volume 2 of OCAMI Stud., pages 163-168. Osaka Munic. Univ. Press, Osaka, 2007.

[41] David Eelbode. Irreducible sl $(m)$-modules of Hermitean monogenics. Complex Var. Elliptic Equ., 53(10):975-987, 2008.

[42] David Eelbode and Fu Li He. Taylor series in Hermitean Clifford analysis. Complex Anal. Oper. Theory, 5(1):97-111, 2011.

[43] Min $\mathrm{Ku}$ and Daoshun Wang. Half Dirichlet problem for matrix functions on the unit ball in Hermitian Clifford analysis. J. Math. Anal. Appl., 374(2):442-457, 2011.

[44] Dixan Peña-Peña, Irene Sabadini, and Frank Sommen. Quaternionic Clifford analysis: the Hermitian setting. Complex Anal. Oper. Theory, 1(1):97-113, 2007.

[45] R. Rocha-Chavez, M. Shapiro, F. Sommen, Integral theorems for functions and differential forms in $\mathbb{C}_{m}$, Research Notes in Math. 428, Chapman\&Hall / CRC (New York, 2002).

[46] Irene Sabadini and Frank Sommen. Hermitian Clifford analysis and resolutions. Math. Methods Appl. Sci., 25(16-18):1395-1413, 2002. Clifford analysis in applications.

[47] F. Sommen. An algebra of abstract vector variables, Portugaliae Mathematica 54, Fasc. 3 (1997), 287-310.

[48] Daniele C. Struppa. Quaternionic and Clifford Analysis in several variables. General aspects of quaternionic and Clifford analysis. Handbook of Operator Theory 